\title{
A Large-Scale Simulation of the Piriform Cortex by a Cell Automaton-Based Network Model
}

\author{
Enric T. Claverol*, Andrew David Brown, Senior Member, IEEE, and John Edward Chad
}

\begin{abstract}
An event-driven framework is used to construct a physiologically motivated large-scale model of the piriform cortex containing in the order of $10^{5}$ neuron-like computing units. This approach is based on a hierarchically defined highly abstract neuron model consisting of finite-state machines. It provides computational efficiency while incorporating components which have identifiable counterparts in the neurophysiological domain. The network model incorporates four neuron types, and glutamatergic excitatory and $G A B A_{A}$ and $G A B A_{B}$ inhibitory synapses.

The spatio-temporal patterns of cortical activity and the temporal and spectral characteristics of simulated electroencephalograms (EEGs) are studied. In line with previous experimental and compartmental work, 1) shock stimuli elicit EEG profiles with either isolated peaks or damped oscillations, the response type being determined by the intensity of the stimuli, and 2) temporally unpatterned input generates EEG oscillations supported by model-wide waves of excitation.
\end{abstract}

Index Terms-Cell automata, discrete simulation, EEG oscillations, piriform olfactory cortex, pulse-coded neuron model.

\section{INTRODUCTION}

$\mathbf{T}$ HE simulation of the mechanisms implicated in information processing in the nervous system is an area of active research [1]-[7]. It provides a tool for the understanding of brain functions which are difficult to study experimentally due to the large number of cells involved and the difficulties arising from the execution of in vivo experiments.

The techniques used for the simulation of large aggregates of neurons can be grouped into two categories: biophysically detailed models [1]; and artificial neural networks [2]. Biophysically detailed models are based on cable theory applied to dendrites and axons and make use of ion channel models which are usually described using the Hodgkin-Huxley formalism [3]. In this context, neurons are described by systems of nonlinear differential equations which must be solved numerically. Two undesirable properties of this approach are the computational cost of numerical integration and the amount of experimental

Manuscript received July 12, 2001; revised April 5, 2002. This work was supported in part by the Biotechnology and Biological Sciences Research Council, U.K., and in part by the Department of Electronics and Computer Science, University of Southampton, U.K. Asterisk indicates corresponding author.

*E. T. Claverol is with the Department of Biology, California Institute of Technology, 1200 California Blvd., Pasadena, CA 91125 USA (e-mail: enric@its.caltech.edu).

A. D. Brown is with the Electronic System Design Group, Electronics Department, Southampton University, SO16 7PX Hampshire, U.K.

J. E. Chad is with the Centre for Neurosciences, Southampton University, SO16 7PX Hampshire, U.K.

Publisher Item Identifier 10.1109/TBME.2002.801986. data required to set the parameters in the model. As a result of these limitations, the simulation of large aggregates of neurons (more than $10^{4}$ ) is unfeasible or requires parallel architectures. During the past decade, several software tools have been developed for the realistic simulation of single cells and small aggregates of neurons (e.g., GENESIS [5] and NEURON [6]). There is also ongoing research to develop simulators capable of handling large networks [4] by means of parallel architectures. At the opposite end of the spectrum, artificial neural networks, in general, do not allow direct mapping of biophysical parameters into model parameters and are considered too unrealistic to be used in physiologically motivated simulation. Their simplicity, however, results in computational efficiency.

We have chosen to extend the approach based on highly abstract neuron-like computing units, typical of the artificial neural networks domain, to the problem of physiologically motivated neural simulation. The underlying assumption is that the inherent biological properties of neurons stabilize their computational function and, hence, abstract single-cell representations may, at the network level, display physiologically realistic behavior while retaining their computational efficiency. To this end, and drawing from the methods used in discrete simulation, an event-driven neuron model can be enriched with physiologically motivated functionality (e.g., synaptic latency, axonal propagation delay, finite refractory period, firing threshold, and others). Such a neuron-like device can be modeled as a complex finite-state machine (an automaton [7]), retaining the efficiency inherent in discrete simulation [8] and making possible large-scale simulations with the available computing resources.

The availability of both experimental data [9], [10] and simulations based on biophysically detailed models [11], [12], makes the piriform cortex an ideal cortical module to validate this approach. The piriform cortex is thought to be involved in smell recognition [13]. It receives input from the olfactory bulb, which performs the first stages in smell identification [14], through the lateral olfactory track (LOT). After carrying out certain computations on the input data (the nature of which is still unclear) it relays its output to higher level cortical modules. Previous simulations have been confined to networks of 4500 neurons in [11] and 292 neurons in [12], far from the approximately $10^{7}$ neurons found in the piriform cortex.

A cortical model is presented in this paper which includes $10^{5}$ discrete neurons of four types: fast glutamate excitatory (cortical pyramidal cells), inhibitory neurons with a preference for $G A B A_{A}$ receptors, inhibitory neurons with a preference 

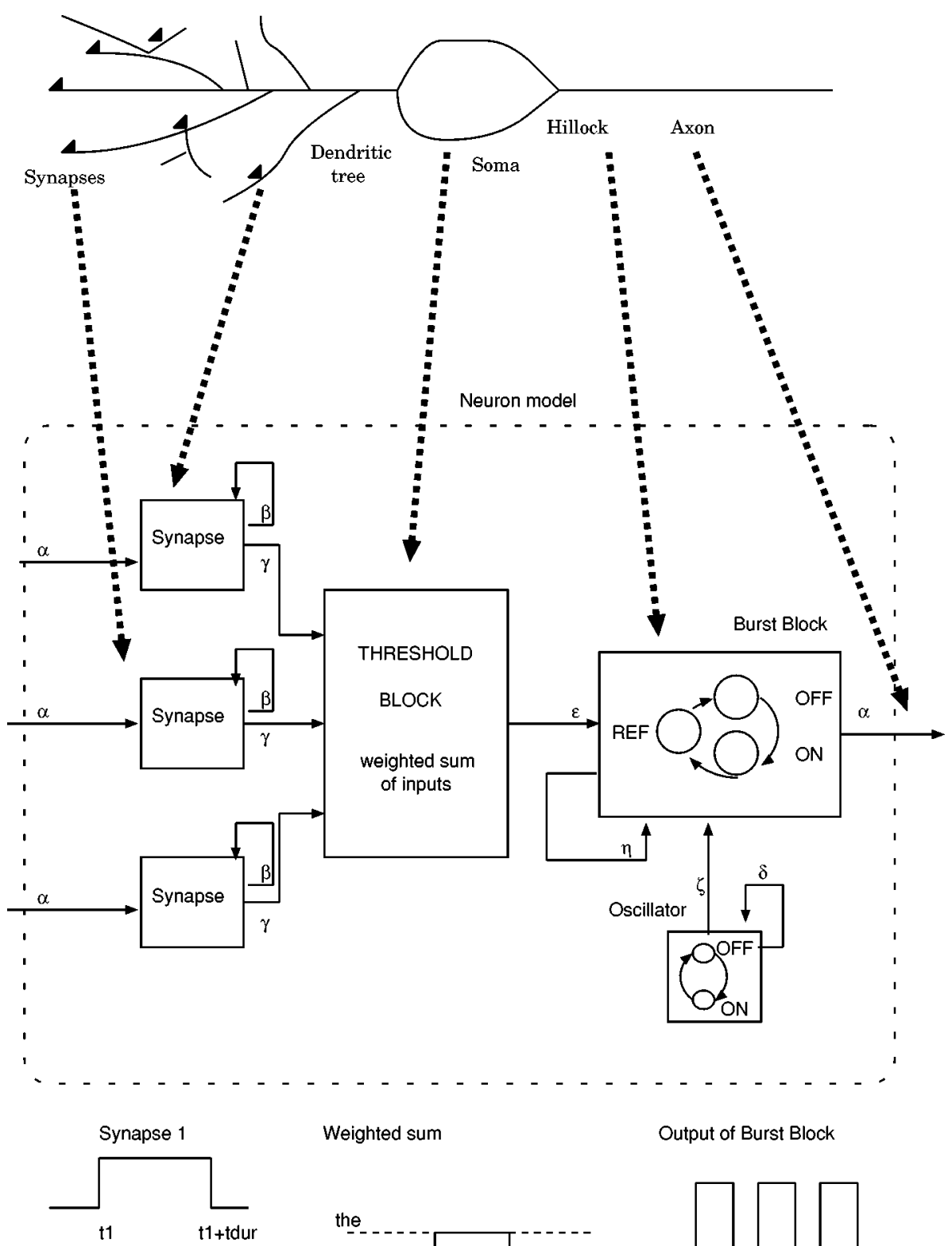

Weighted sum

Output of Burst Block
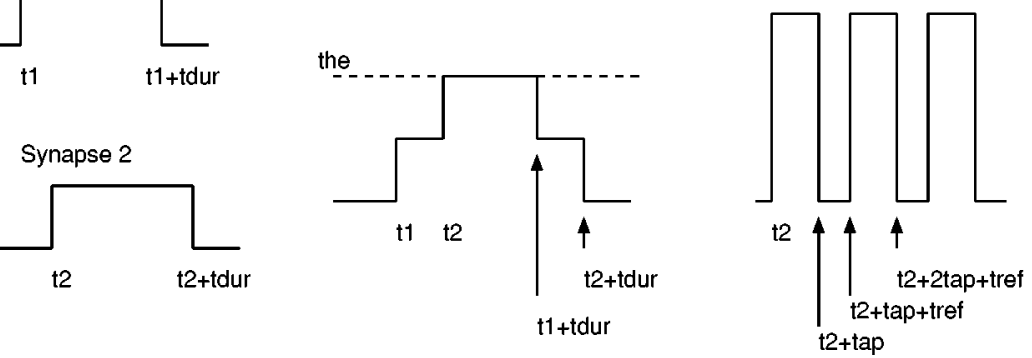

Fig. 1. Message-based event-driven neuron model. Solid arrows indicate origins and destinations of messages. Thick dashed arrows indicate the correspondence between parts of the real neuron and blocks in the model.

for slow $G A B A_{B}$ receptors, and mitral neurons. The message-based event-driven neuron model will be described first. Second, the piriform cortex model and the calculations involved in the estimation of field potentials and electroencephalograms (EEGs) will be discussed. Third, the responses of the cortical model to shock stimulus and random input will be studied and shown to share the main characteristics of experimental data and results obtained with compartmental models. Issues regarding the implementation of an efficient simulator for this type of models and networks in the order of $10^{5}$ neurons are discussed elsewhere [15].

\section{METHODS}

\section{A. Message-Based Event-Driven Neuron Model}

The message-based event-driven neuron model is a hierarchically defined finite-state automaton ${ }^{1}$ [16]. It is made up of several blocks, each of them capturing the functionality of a different component of the neuron (see Fig. 1).

Message passing is the method used for communication between model neurons and between blocks within a single neuron. Each message is a data packet containing the time at

${ }^{1}$ Available: www.its.caltech.edu/ enric/TBME 
TABLE I

Message ChanNels in the Neuron Model

\begin{tabular}{cccc}
\hline \hline Channel & Message structure & $\begin{array}{c}\text { Legal values } \\
\text { of } \mathrm{m}\end{array}$ & $\begin{array}{c}\text { Legal values } \\
\text { of } \mathrm{p}\end{array}$ \\
\hline$\alpha$ & $\{\mathrm{t}, \mathrm{m}\}$ & on & \\
$\beta$ & $\{\mathrm{t}, \mathrm{m}\}$ & on,off & \\
$\gamma$ & $\{\mathrm{t}, \mathrm{m}, \mathrm{p}\}$ & on,off & efficacy $\left(w_{\text {syn }}\right)$ \\
$\delta$ & $\{\mathrm{t}, \mathrm{m}\}$ & change & \\
$\epsilon$ & $\{\mathrm{t}, \mathrm{m}\}$ & on,off & \\
$\zeta$ & $\{\mathrm{t}, \mathrm{m}\}$ & on & \\
$\eta$ & $\{\mathrm{t}, \mathrm{m}\}$ & off,$r_{-}$off & \\
\hline
\end{tabular}

TABLE II

PARAMETERS IN THE NEURON MODEL

\begin{tabular}{cl}
\hline \hline Parameter & \multicolumn{1}{c}{ Function } \\
\hline$t h_{e}$ & Excitation threshold \\
$t h_{i}$ & Inhibition threshold \\
$t_{a p}$ & Duration of action potential \\
$t_{r e f}$ & Duration of refractory period \\
$N_{b u r s t}$ & Number of spikes per burst \\
$t_{\text {osc }}$ & Period of pace maker \\
$t_{\phi}$ & Time offset of pace maker \\
$t_{d e l}$ & Synaptic delay \\
$t_{d u r}$ & Duration of synaptic pulse \\
$w_{s y n}$ & Synaptic efficacy \\
\hline
\end{tabular}

which the message will be delivered to its destination, a label field indicating the type of message and a third optional field with extra information used by the target neuron to process the message (see Table I). Arrows with solid lines in Fig. 1 indicate message paths.

The delivery of a message to a block, triggers the update of its state, which may be accompanied by the broadcasting of new messages (an output) and the update of state variables in the block (an action). For purely combinational functions (e.g., the synapse block), the output is only a function of the input.

Table II lists the parameters required for the configuration of each neuron and synapse in the model (different neuronal and synaptic types are conferred different functional characteristics by setting these parameters). Tables III-VI are the state-transition tables and combinational functions implemented by the blocks in the neuron model.

1) The Synapse Block: Synapses receiving the on message at time $t$, which notifies of the firing of a presynaptic neuron, introduce a synaptic delay and become activated at $t+t_{\text {del }}$. An on message is then broadcast to the threshold block. At $t+t_{\mathrm{del}}+t_{\mathrm{dur}}$, the synapse inactivates, having remained activated for $t_{\text {dur }}$ time units, and sends an off message to the threshold block. Synapses are combinational functions which schedule new messages depending on the last message received (they do not need memory of their current state).

2) The Threshold Block: The threshold block computes a weighted sum of inputs $\left(w_{\text {sum }}\right)$ where the weights are the synaptic efficacies $\left(w_{\text {syn }}\right)$. The arrival of on and off messages from synapses, triggers the update of $w_{\text {sum }}$. After an update, its value is compared against the excitation $\left(t h_{e}\right)$ and inhibition $\left(t h_{i}\right)$ thresholds. An on message is sent to the burst generator block (which generates a burst of action potentials) if $w_{\text {sum }}$
TABLE III

THE SyNAPSE BLOCK FUNCTION

\begin{tabular}{c|c}
\hline \hline \multicolumn{2}{|c}{ Synapse block } \\
\hline Input & Output \\
\hline$\alpha:=$ on & $\beta:=\left\{t_{\text {del }}\right.$, on $\}$ \\
\hline$\beta:=$ on & $\beta:=\left\{t_{\text {dur }}\right.$, off $\}, \gamma:=\left\{0\right.$, on,$\left.w_{\text {syn }}\right\}$ \\
\hline$\beta:=$ off & $\gamma:=\left\{0\right.$, of $\left.f,-w_{\text {syn }}\right\}$ \\
\hline
\end{tabular}

TABLE IV

THe THReshold Block StATE Machine

\begin{tabular}{c|c}
\hline \hline \multicolumn{2}{c}{ Threshold block } \\
\hline Input & Action $\mid$ Output \\
\hline \multirow{5}{*}{$\gamma:=$ on } & $w_{\text {sum }}+=w_{\text {syn }}$ \\
& $w_{\text {sum }}>=t h_{e}$ \\
& true: $-\mid \epsilon:=\{0$, on $\}$ \\
& $w_{\text {sum }}<=t h_{i}$ \\
& true: $-\mid \epsilon:=\{0$, off $\}$ \\
\hline \multirow{5}{*}{$\gamma:=$ off } & $w_{\text {sum }}-=w_{\text {syn }}$ \\
& $w_{\text {sum }}>=t h_{e}$ \\
& true: $-\mid \epsilon:=\{0$, on $\}$ \\
& $w_{\text {sum }}<=t h_{i}$ \\
& true: $-\mid \epsilon:=\{0$, off $\}$ \\
\hline
\end{tabular}

TABLE V

The OsCillator State Machine

\begin{tabular}{c|c}
\hline \hline \multicolumn{2}{|c}{ Oscillator } \\
\hline Current state & Next state $\mid$ Output \\
\hline & Input \\
\hline & $\delta:=\left\{t_{\text {osc }}\right.$, change $\}$ \\
\hline on & off $\mid \delta:=\left\{t_{\text {osc }}\right.$, change $\}, \zeta:=\{0$, on $\}$ \\
\hline off & on $\mid \delta:=\left\{t_{\text {osc }}\right.$, change $\}, \zeta:=\{0$, on $\}$ \\
\hline
\end{tabular}

increases beyond $t h_{e}$. Conversely, if the weighted sum becomes more negative than the inhibition threshold $\left(t h_{i}\right)$, the threshold block sends an off message to the burst block to stop an ongoing burst. Note that, since neurons in the cortical model presented in this paper were configured to fire single spikes, burst truncation does not apply and it was disabled, in order to avoid the unnecessary generation of off messages, by setting $t h_{i}$ to -1000 , a value never reached by $w_{\text {sum. }}$. Therefore, the possibility of burst truncation remained hardwired in the model to support future studies including bursting neurons but was disabled for the simulations discussed here.

3) The Oscillator Block: The oscillator block simulates rhythmic activity in neurons. It sends an on message to the burst generator block every $t_{\mathrm{Osc}}$ time units starting at $t=t_{\phi}$.

4) The Burst Generator Block: The burst generator creates action potentials as follows: the arrival of the on message triggers a change from state off to state on (onset of the first action potential). After $t_{a p}$ time units, the state changes from on to ref (beginning of the refractory period). After $t_{\text {ref }}$ time units it returns to the initial state off. An on message is broadcast to all postsynaptic cells driven by the burst block whenever its state changes to $o n$.

In the presented cortical model, neurons fire single action potentials upon integration of sufficient $\left(w_{\mathrm{sum}}>t h_{e}\right)$ synaptic excitation. However, to support future network models with 
TABLE VI

THE BURST GENERATOR STATE MAChine

\begin{tabular}{|c|c|c|c|c|c|}
\hline \multicolumn{6}{|c|}{ Burst generator } \\
\hline \multirow{3}{*}{$\begin{array}{c}\text { Current } \\
\text { state } \\
\end{array}$} & \multicolumn{5}{|c|}{\begin{tabular}{|l|l|l|} 
Next state / Action & Output \\
\end{tabular}} \\
\hline & \multicolumn{5}{|c|}{ Input } \\
\hline & $\epsilon:=o n$ & $\epsilon:=$ off & $\eta:=$ off & $\eta:=r_{-} o f f$ & $\zeta:=$ on \\
\hline on & on $1-1-$ & on $\left|n_{\text {burst }}=0\right|-$ & \begin{tabular}{r|l|l}
$\operatorname{ref}$ & $\eta:=\left\{t_{r e f}, r_{-} o f f\right\}$ \\
\end{tabular} & \begin{tabular}{ll|l} 
on & $-1-$ \\
\end{tabular} & on $\mid-1-$ \\
\hline ref & $\operatorname{ref} \mid-1-$ & ref $\left|n_{\text {burst }}=0\right|-$ & ref $1-1-$ & $\begin{array}{c}n_{b u r s t}-1=0 \text { ? } \\
\text { true: off }\left|n_{b u r s t}=N_{\text {burst }}\right|- \\
\text { false: } \\
\text { on }\left|n_{b u r s t}-1\right| \alpha:=\{0, \text { on }\} \\
\eta:=\left\{t_{a p}, \text { off }\right\}\end{array}$ & $\operatorname{ref} \mid-1-$ \\
\hline off & $\begin{aligned} \text { on } \mid & -\alpha:=\{0, \text { on }\}, \\
\eta & :=\left\{t_{\text {ap }}, \text { off }\right\}\end{aligned}$ & off $|-|-$ & off $|-|-$ & off $\mid-1-$ & $\begin{array}{c}\text { on }|-| \alpha:=\{0, \text { on }\}, \\
\eta:=\left\{t_{\left.a_{p}, \text { off } f\right\}}\right.\end{array}$ \\
\hline
\end{tabular}

TABLE VII

Numerical VALUes of PARAMETERS IN THE Piriform CorteX MOdei

\begin{tabular}{|c|c|}
\hline \multicolumn{2}{|c|}{ Neuronal parameters } \\
\hline the (pyramidal) & 7 \\
\hline the (fast inh.) & 30 \\
\hline$t h{ }_{e}$ (slow inh.) & 30 \\
\hline$t h_{i}$ & -1000 (burst truncation inactivated) \\
\hline$t_{a p}$ & $1 \mathrm{~ms}$ \\
\hline$t_{r e f}$ & $10 \mathrm{~ms}$ \\
\hline$N_{\text {burst }}$ & 1 \\
\hline$t_{o s c}$ (pyramidals and inhibitory) & 0 (inactive oscillator) \\
\hline$t_{\phi}$ (pyramidals and inhibitory) & 0 (inactive oscillator) \\
\hline$t_{\text {osc }}$ (LOT axons, all stimuli) & $3000 \mathrm{~ms}$ \\
\hline$t_{\phi}$ (LOT axons, shock stimulus) & $0 \mathrm{~ms}$ \\
\hline$t_{\phi}$ (LOT axons, random input) & Uniform $\left(0-t_{\text {stop }}\right)$ \\
\hline \multicolumn{2}{|c|}{ Number of synapses per neuron } \\
\hline LOT to pyramidal & 100 \\
\hline pyramidal to pyramidal & 300 \\
\hline pyramidal to fast inhibitory & 20 \\
\hline pyramidal to slow inhibitory & 10 \\
\hline fast inhibitory to pyramidals & 70 \\
\hline slow inhibitory to pyramidals & 60 \\
\hline \multicolumn{2}{|c|}{ Synaptic parameters } \\
\hline$t_{\text {del }}$ (pyramidal to pyr./inh.) & $(3-12 m s)$ \\
\hline$t_{d u r}$ (pyramidal to pyr./inh.) & $5 \mathrm{~ms}$ \\
\hline$w_{s y n}$ (pyramidal to pyr./inh.) & 1 \\
\hline$t_{\text {del }}$ (fast inh. to pyramidal) & $5 \mathrm{~ms}$ \\
\hline$t_{d u r}($ fast inh. to pyramidal) & $12 \mathrm{~ms}$ \\
\hline$w_{s y n}$ (fast inh. to pyramidal) & -15 \\
\hline$t_{d e l}$ (slow inh. to pyramidal) & $10 \mathrm{~ms}$ \\
\hline$t_{d u r}$ (slow inh. to pyramidal) & $150 \mathrm{~ms}$ \\
\hline$w_{\text {syn }}$ (slow inh. to pyramidal) & -1 \\
\hline$t_{\text {del }}(\mathrm{LOT}$ to pyramidal $)$ & $(1-4 m s)$ \\
\hline$t_{d u r}$ (LOT to pyramidal) & $5 m s$ \\
\hline$w_{\text {syn }}$ (LOT to pyramidal) & 4 \\
\hline \multicolumn{2}{|c|}{ Mean connection range, $\lambda$ (normalized distance) } \\
\hline pyramidal to pyramidal & 2 \\
\hline pyramidal to fast inhibitory & 10 \\
\hline pyramidal to slow inhibitory & 10 \\
\hline fast inhibitory to pyramidals & 10 \\
\hline slow inhibitory to pyramidals & 10 \\
\hline LOT to pyramidals & 2 \\
\hline
\end{tabular}

bursting neurons, the proposed neuron model includes a (more general) burst generator block capable of creating bursts with $N_{\text {burst }}$ spikes ( $N_{\text {burst }}=1$ in this paper). ${ }^{2}$

\section{B. Piriform Cortex Model}

This discrete model of the piriform cortex is based on the compartmental simulations by Wilson et al. [11] and Barkai

\footnotetext{
${ }^{2}$ The sequence of state transitions during generation of bursts $\left(N_{\text {burst }}>1\right)$ differs with respect to those for single spikes $\left(N_{\text {burst }}=1\right)$, at the end of the first refractory period. In bursts, the end of the first refractory period triggers a state transition to on (start of the second action potential in the sequence) and the cycle is repeated $N_{\text {burst }}$ times, making up a burst of $N_{\text {burst }}$ action potentials.
}

et al. [12]. Four types of cells have been included (Fig. 2): fast excitatory pyramidal cells, fast inhibitory $\left(G A B A_{A}\right)$ cells, slow inhibitory $\left(G A B A_{B}\right)$ cells and mitral olfactory bulb cells, which provide stimulus to the cortex and whose axons form the LOT.

The pyramidal cell layer consists of a grid of $250 \times 250$ neurons whereas $G A B A_{A}$ and $G A B A_{B}$ inhibitory cells are arranged in two layers of $80 \times 80$ cells each. For clarity, these are depicted in separate planes in Fig. 2. However, they occupy the same plane in the actual model.

The top-layer models the input activity that arrives at the piriform cortex from the mitral cells in the olfactory bulb carried by the LOT. The number of cells in this pool has been adjusted for each simulation in order to provide the desired rate of excitation. As they are topologically far from the rest of the cells, these neurons do not contribute to the simulated field potential recordings.

Pyramidal cells possess local and long range intralayer excitatory connections (amongst pyramidal cells) and local interlayer connections (exciting nearby $G A B A_{A}$ and $G A B A_{B}$ cells). Inhibitory cells ( $G A B A_{A}$ and $G A B A_{B}$ layers) do not have intralayer connections in this model. Instead, they locally inhibit pyramidal cells by means of local connections.

The target neuron, $j$, of a synapse from neuron $i$ is chosen as the closest cell to the position given by a random vector $\vec{d}$ of components $\{\rho, \phi\}$ (in polar coordinates), where $\rho$ is an exponential random variable (mean given in Table VII) and $\phi$ is a uniform variable in the range $0-2 \pi$. The target neuron is chosen as the closest cell to the vector

$$
\overrightarrow{p_{j}}=\overrightarrow{p_{i}}+\vec{d}
$$

where $\overrightarrow{p_{i}}$ and $\overrightarrow{p_{j}}$ are the position vectors for neurons $i$ and $j$ respectively.

LOT axons synapse onto pyramidal cells and introduce external stimulus into the model. The density of connections from LOT to pyramidal cells decreases exponentially from left to right (rostral to caudal in the real cortex) in the pyramidal layer of Fig. 2.

Any one synapse in the model is completely characterized by a synaptic parameter triplet $\left(t_{\mathrm{del}}, t_{\mathrm{dur}}, w_{\mathrm{syn}}\right)$. The duration of the synaptic activation $\left(t_{\mathrm{dur}}\right)$ was initially set to half-maximum values of typical postsynaptic potentials and later adjusted to achieve realistic shock and random stimulus responses. The final values were $5 \mathrm{~ms}$ for excitatory synapses and $12 \mathrm{~ms}$ and $150 \mathrm{~ms}$ for $G A B A_{A}$ and $G A B A_{B}$ synapses respectively. 


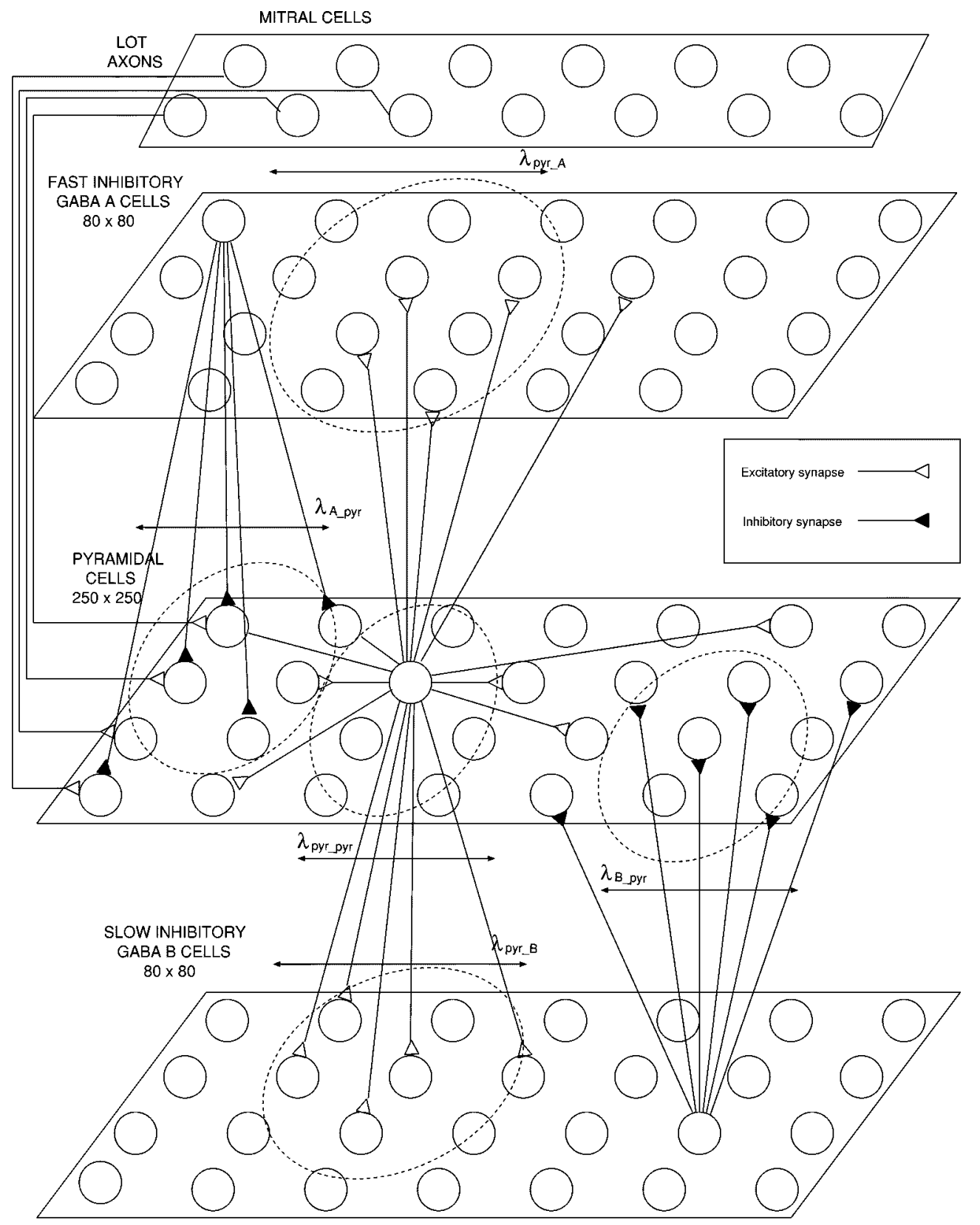

Fig. 2. Model of the piriform cortex.

The synaptic delay parameter $\left(t_{\mathrm{del}}\right)$ accumulates presynaptic axonal propagation delay and onset to peak latency of the postsynaptic potentials. For LOT to pyramidal and pyramidal-pyramidal connections, the axonal delay was calculated from action potential propagation velocities in LOT axons $(1.6 \mathrm{~m} / \mathrm{s})$ and in pyramidal cell axons $(0.6 \mathrm{~m} / \mathrm{s}$, the average of the velocities for rostrally and caudally directed fibers used by Wilson et al. [11]). Further, to reduce memory consumption, the delay introduced by long-range pyramidal cell axons and LOT afferents was quantised and the number of allowed values limited to 10 and 4 respectively. Similarly, the delay introduced by the axons of inhibitory cells, given the local nature of these connections compared to the long-range pyramidal-pyramidal axons, was assumed distance independent (see Table VII). Overall, the quantization of axonal delays, reduces the number of different synaptic parameter triplets to 16 , allowing a 4-bit representa- tion of the synaptic configuration, a strategy that minimizes the memory space necessary to store the model. ${ }^{3}$

The synaptic efficacy $\left(w_{\text {syn }}\right)$ was arbitrarily set to 1 for excitatory synapses and to -1 and -15 for slow and fast inhibitory synapses, respectively, mantaining the relative efficacy as indicated by the maximal conductance of synaptic conductances used in previous compartmental models.

Neurons fire a single action potential (with a duration of $1 \mathrm{~ms}$ and followed by a $10-\mathrm{ms}$ refractory period) whenever $w_{\text {sum }}$ (weighted sum of inputs) increases above the excitation

\footnotetext{
${ }^{3}$ The quantization of delays causes artifacts in shock stimulus simulations consisting in precisely delimited cortical bands showing homogeneous neuronal states (Fig. 7, $t=13 \mathrm{~ms}$ and Fig. 8, $t=14 \mathrm{~ms}$ ). This effect is not present in EEG simulations with more realistic (random) stimuli [e.g., Fig. 9(c)], for which reason the computational advantages of delay quantization motivated the introduction of this simplification in the model.
} 


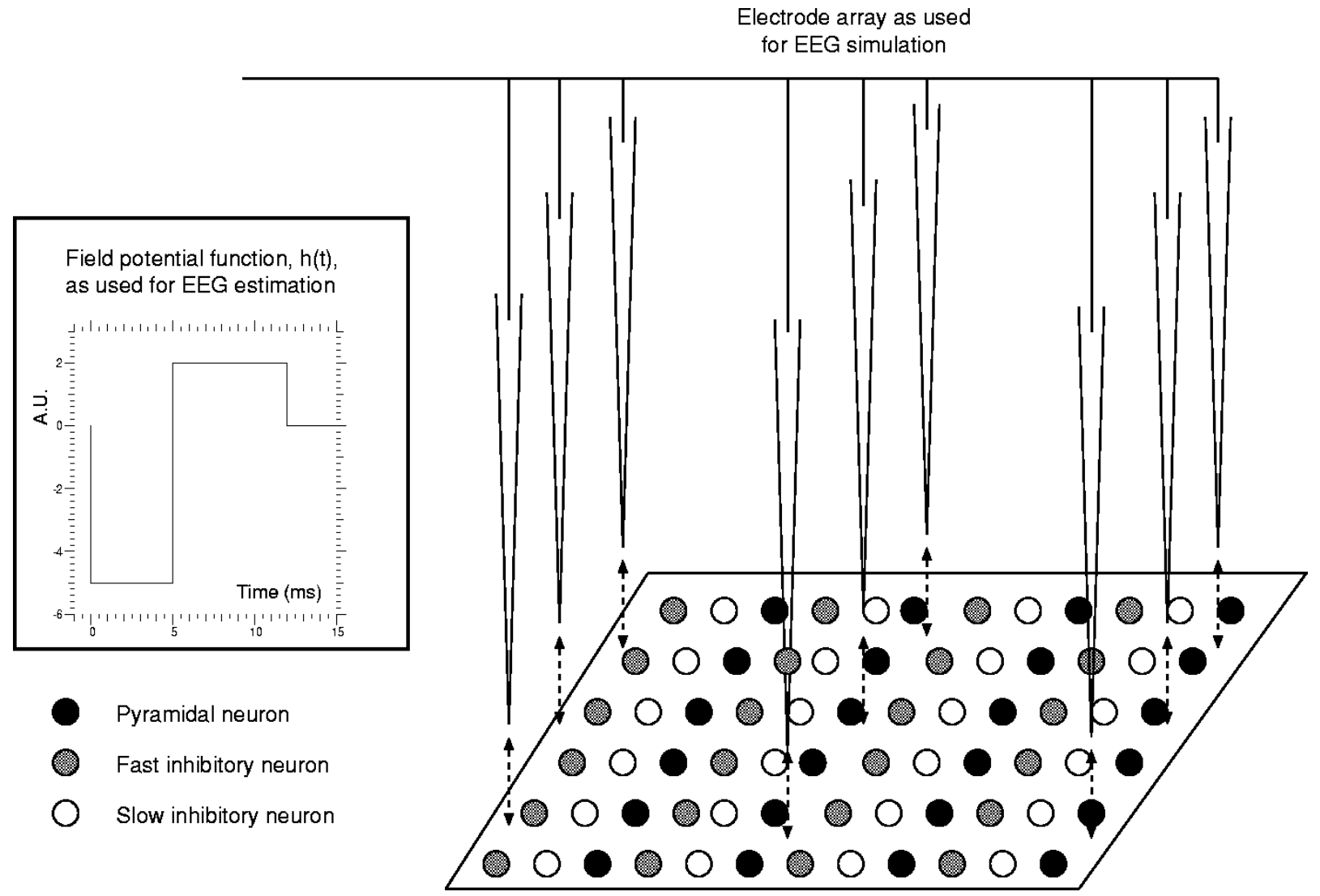

Fig. 3. Setup used for the simulation of field recordings and EEGs.

threshold. Suitable values for the excitation threshold were determined by parameter space search.

A complete list of numerical values for the parameters of the model are provided in Table VII.

\section{Simulation of Field Potential Recordings, EEGs and Power Spectra}

Field potentials and EEGs are measurements of time changing potentials generated by neuronal activity. Field potentials are recorded with single microelectrodes located close to the pool of neurons under study whereas EEGs are recorded with electrodes placed on the skull. For the purpose of model validation, it is desirable to compare the characteristics of the recordings predicted by the model with those seen in experimentally recorded signals.

For the simulation of EEG recordings, a procedure similar to that described by Wilson et al. [11] has been followed. A number of virtual electrodes are spatially distributed forming a grid of $E \times E$ recording sites (Fig. 3). Each one of these simulated electrodes obtains a field potential (FP) calculated as

$$
S_{\mathrm{FP}_{\mathrm{i}}}=\sum_{j}^{J} \sum_{k}^{K} \frac{1}{d_{i j}} \delta_{j}\left(t-t_{k}\right) * h(t)
$$

where $S_{\mathrm{FP}_{\mathrm{i}}}$ is the field potential signal recorded by the $i$ th electrode, $d_{i j}$ is the distance between the $i$ th electrode and neuron $j, \delta_{j}\left(t-t_{k}\right)$ is the delta function indicating that neuron $j$ fired an action potential at $t=t_{k}$ and $h(t)$ is the prototype field potential recorded from a group of neurons firing nearly simulta- neously. The summations are over the total number of action potentials $K$ generated by neuron $j$ and over all the neurons $J$ in the network. The case $d_{i j}=0$ is avoided by positioning the array of electrodes at a plane slightly above the cell layers, so that the minimum electrode-neuron distance is equivalent to the distance between two adjacent pyramidal cells.

The prototype field potential, $h(t)$, is shown in the box in Fig. 3 and given by $(t$ in milliseconds

$$
h(t)= \begin{cases}t<0 & 0 \\ 0<t<5 & -5 \\ 5<t<12 & 2 \\ 12 t & 0\end{cases}
$$

where the negative segment accounts for the negative field potential recorded experimentally during the onset of action potentials and the positive segment corresponds to the positive field potential seen during repolarization (its return to resting voltage) [17], [18].

The EEG signal is obtained by linear combination of the field potentials

$$
S_{\mathrm{EEG}}=\sum_{i}^{E \times E} S_{\mathrm{FP}_{\mathrm{i}}}
$$

where $S_{\mathrm{EEG}}$ is the EEG signal, $S_{\mathrm{FP}_{\mathrm{i}}}$ the field potential recorded by the $i$ th electrode and the summation is over all the electrodes forming the $E \times E$ grid.

For the estimation of the EEG power spectrum, the procedure described in [19] (with Hamming window, segments of 


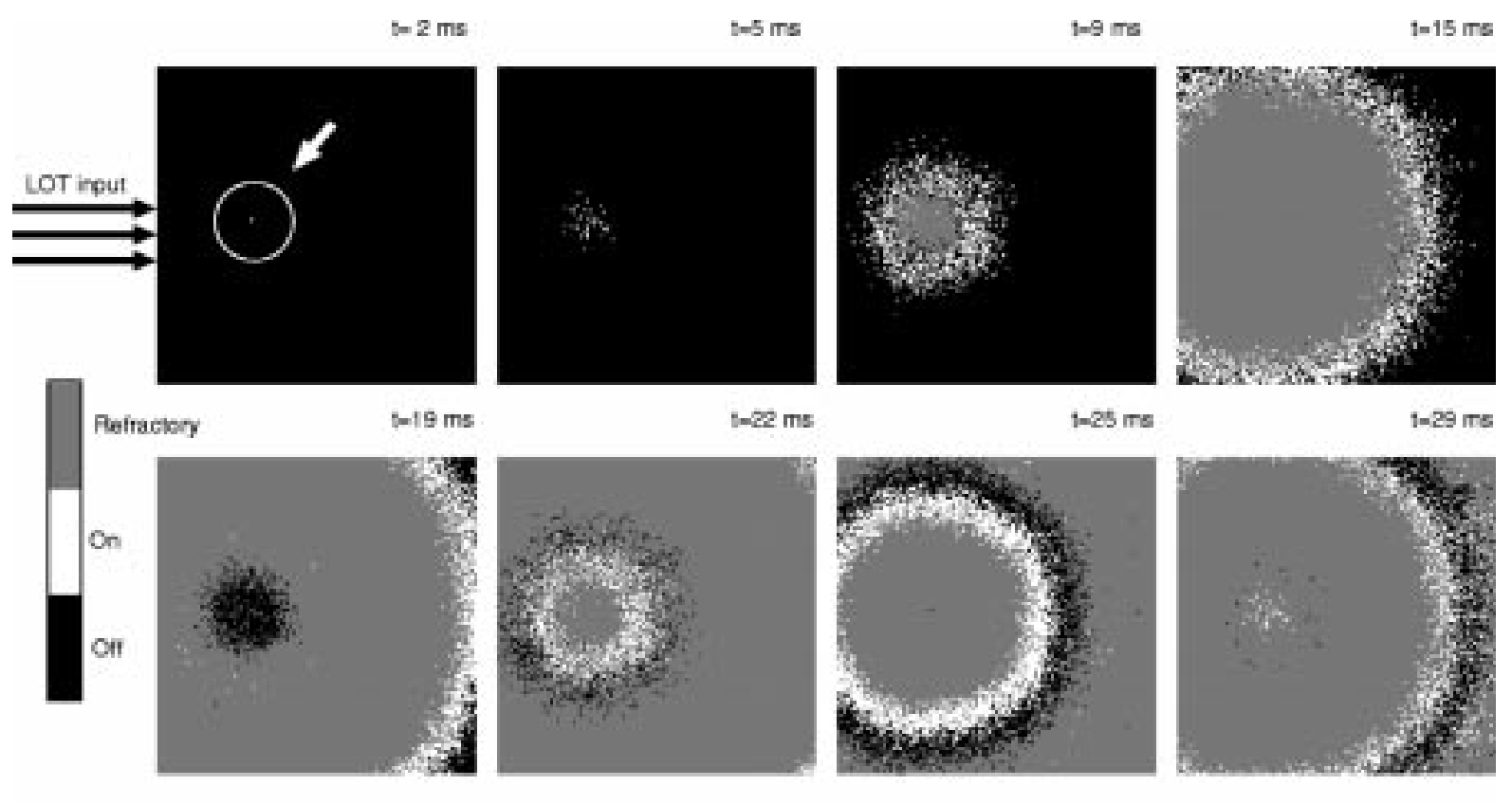

(is)
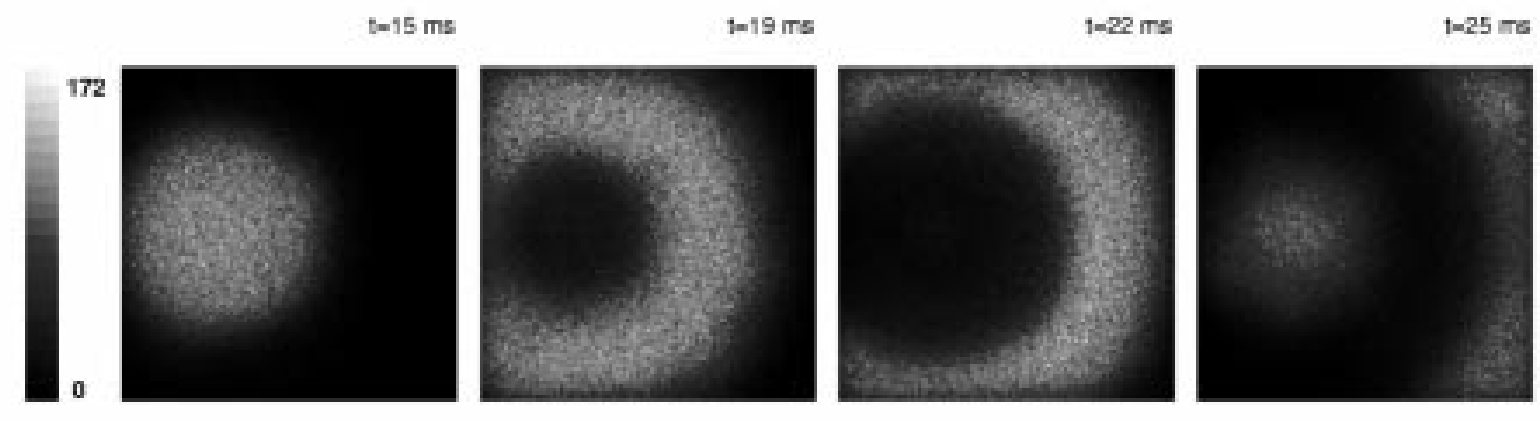

(b)

Fig. 4. Sequence of images representing (a) the state and (b) the weighted sum of inputs $\left(w_{\text {sum }}\right)$ of pyramidal neurons in a partially connected model (each pixel in the arrays corresponds to an individual neuron).

512 samples, half-periodogram overlapping and $1 \mathrm{kHz}$ effective sampling rate) was used.

\section{Computer Resources}

All simulations were run on a 350-MHz desktop PC computer running Linux (kernel version 2.2.5-15) with 320 Mbytes of RAM. The average CPU time taken by $1 \mathrm{~ms}$ of simulation was $0.9 \mathrm{~s}$ in the case of random input stimulus where the network displays realistic behavior and continuous activity throughout the entire simulation. In the case of shock stimulus, activity was restricted to the first $100 \mathrm{~ms}$ and no CPU time was taken by the simulation beyond this point. By reducing the amount of collected data, the number of neurons in the model could be increased by approximately $20 \%$.

\section{RESULTS}

\section{A. Wave Generation in a Partially Connected Model}

To study the mechanisms underlying the continuous generation and propagation of waves in the pyramidal cell layer, the model was simplified first by removing the inhibitory connections from GABA to pyramidal cells and by collapsing the pool of LOT axons into a single unit with connections to one randomly chosen pyramidal neuron. Fig. 4(a) shows an array representation of the state of the pyramidal cells at selected times (black, gray or white pixels correspond to neurons in state off, refractory and on, respectively). Fig. 4(b) shows the value of the weighted sum of inputs $\left(w_{\text {sum }}\right)$, which allows quantification of the total synaptic input received by any one neuron.

At $t=0$, the LOT axon fires an action potential (not shown in the figure), whose excitation reaches its target neuron at $t=$ $2 \mathrm{~ms}$ (see lefmost top panel). At $t=5 \mathrm{~ms}$, local pyramidal to pyramidal connections have started spreading the excitation. At $t=9 \mathrm{~ms}$, the cells located at the core of the patch excited first have become refractory, shown as a transition to a gray shade. At $t=19 \mathrm{~ms}$, the wave reaches the borders of the cortex while most pyramidal cells remain in refractory state. However, those that fired first during the wave, have finished their refractory period and enter the off state. At $t=22 \mathrm{~ms}$, they are re-excited by long range axonal connections carrying action potentials from the distant wave (now at the boundary of the cortex) toward the core. In this way, a second wave is initiated. A similar sequence of events takes place in the simulations of the cortical response to various types of stimuli described below. 
Strong stimulus

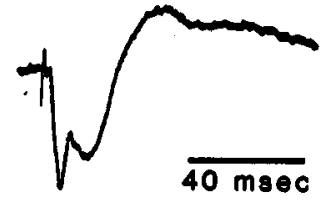

Compartmental simulation by

Wilson and Bower

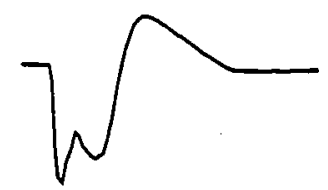

Weak stimulus
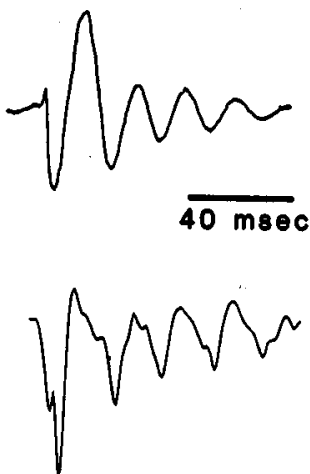

EEG
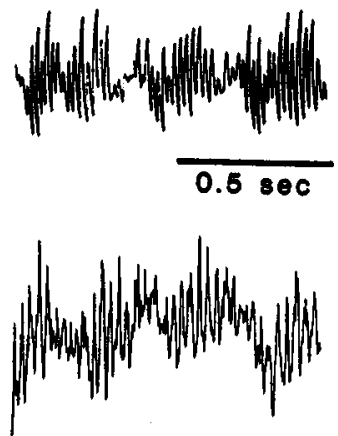

Fig. 5. Recordings (top) and compartmental simulations (bottom) of field potentials after weak shock (left), strong shock (center) and random stimulus (right). Figure reproduced from [11].

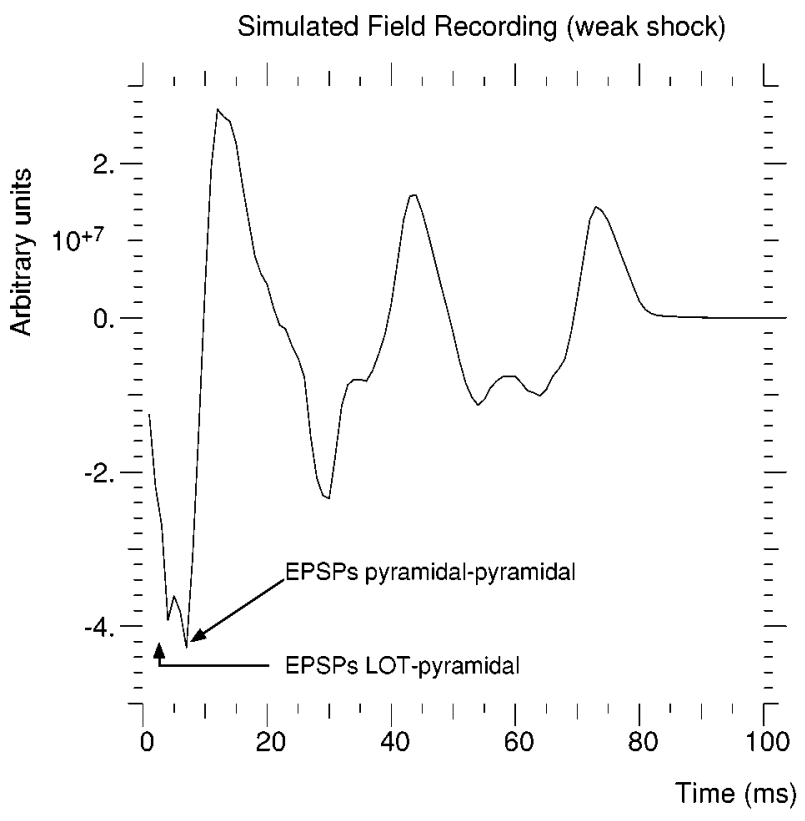

(a)

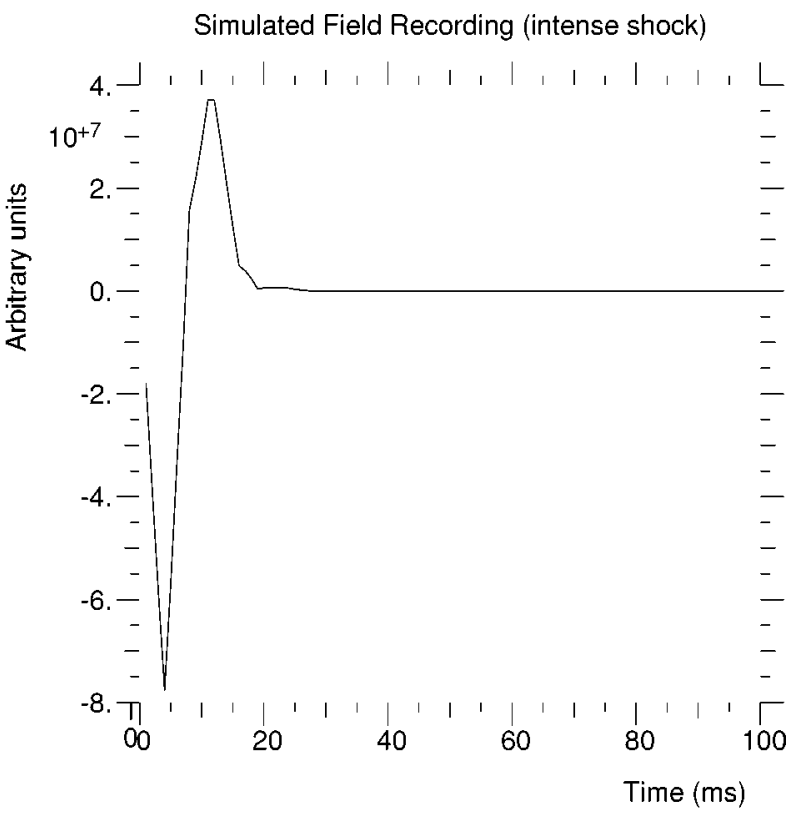

(b)

Fig. 6. (a) Simulated field potential after weak shock stimulus. (b) Simulated field potential after strong shock stimulus.

Comparison of Fig. 4(a) and 4(b) indicates that the finite axonal propagation velocity introduces a delay in the propagation of excitation between pyramidal cells. For instance, although cells are firing in the outer shell at $t=15 \mathrm{~ms}$, the maximal excitatory input is being received at the core [see first panel in 4(b)] where the neurons have already entered the refractory period and are unable to fire.

In this partially connected model, the absence of inhibition allows continuous wave generation with unrealistically regular spatial patterns. The addition of inhibitory connections, described in Sections III-B and C, limits the number and intensity of the waves and introduces irregularity in the wave edges.

\section{B. Shock Stimulus Response}

In shock stimulus experiments, the LOT is stimulated with a short duration current pulse (duration $<1 \mathrm{~ms}$ ) and the response of the olfactory cortex is monitored with a single electrode measuring field potentials. Experimental recordings [20] and comparmental simulations [11] of this experimental paradigm fall in two categories: single wave response and damped oscillation (Fig. 5). High-intensity current pulses stimulate a single peak whereas, counter-intuitively, lower intensity pulses produce long-lasting responses consisting of several damped peaks.

In our model, shock stimulus is generated by the simultaneous firing of all LOT axons at $t=0$. The intensity of the shock is adjusted by changing the total number of cells in the LOT pool. Fig. 6 shows the field potential measured by a single electrode positioned at the center of the cortical layer. In accordance with experimental observations, a weak stimulus generated by 1000 LOT axons generates a damped oscillation [see Fig. 6(a)] whereas an intense stimulus created by 6000 LOT axons triggers a single peak response [shown in Fig. 6(b)].

Understanding of the mechanisms underlying these two responses can be gained studying the panels shown in Figs. 7 and 8 (only pyramidal cells shown). Images correspond to the state (leftmost column), value of $w_{\text {sum }}$ (middle column) and partial contribution to $w_{\text {sum }}$ by other pyramidal cells (rightmost column). 

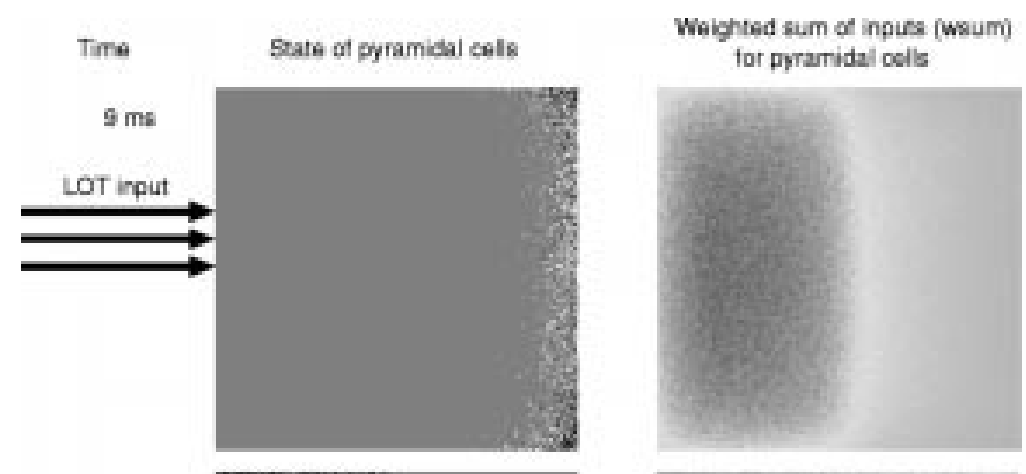

$13 \mathrm{~ms}$

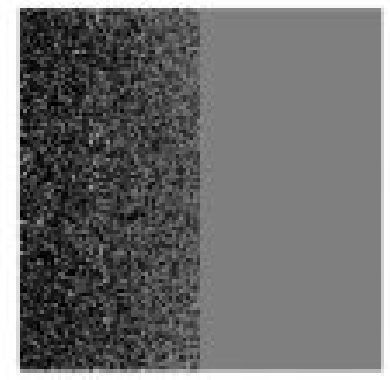

$16 \mathrm{~ms}$

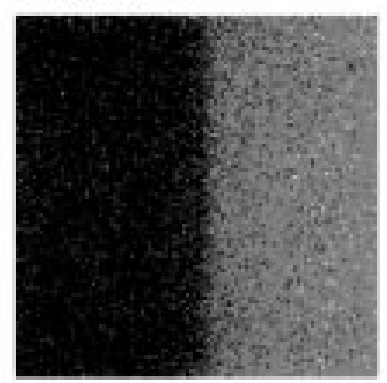

$21 \mathrm{~ms}$

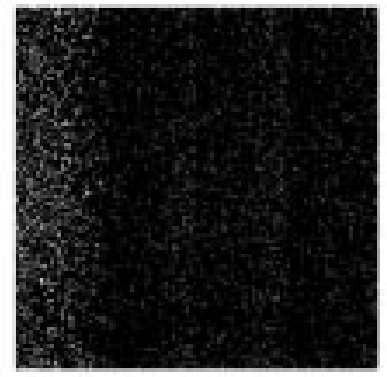

$29 \mathrm{~ms}$

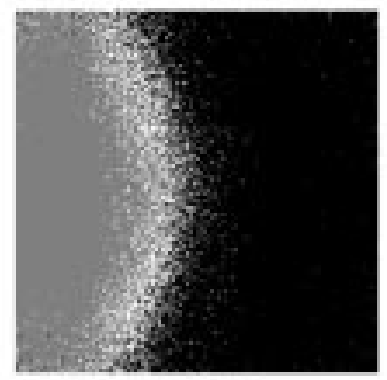

$39 \mathrm{~ms}$

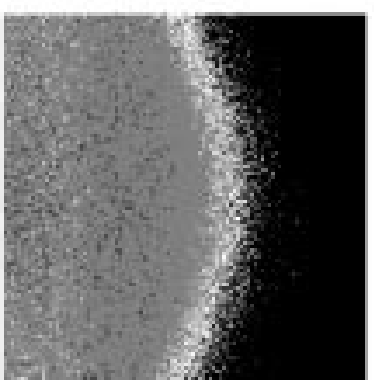

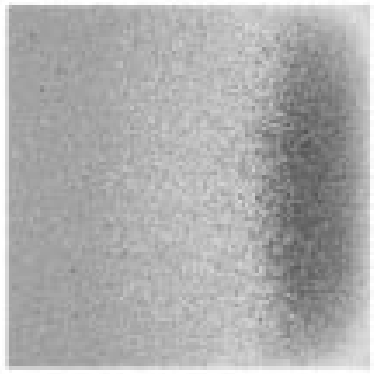
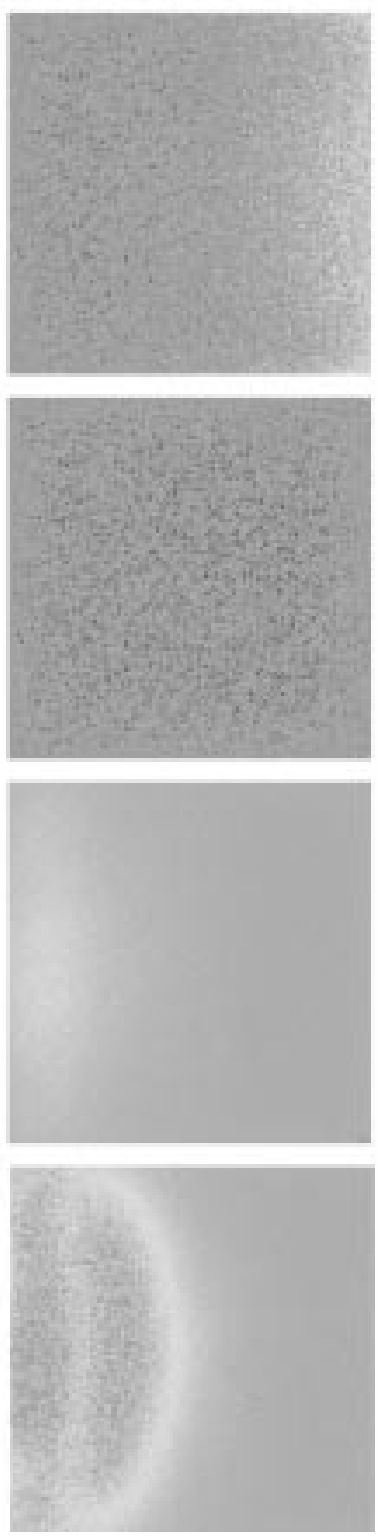

Contribiron of pyramal cels

to esum

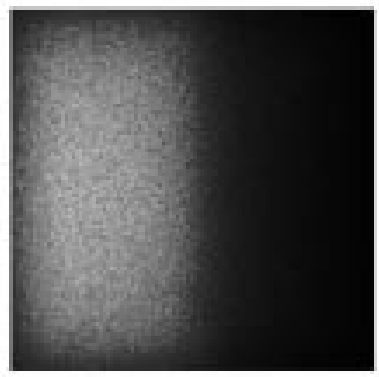

State
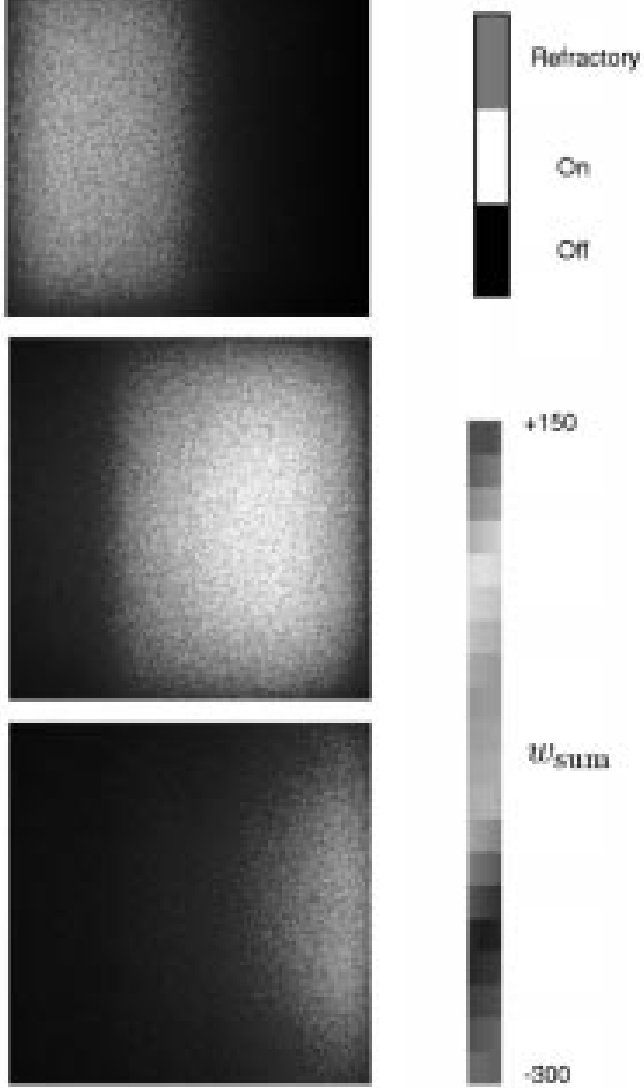

$u_{\text {sum }}$

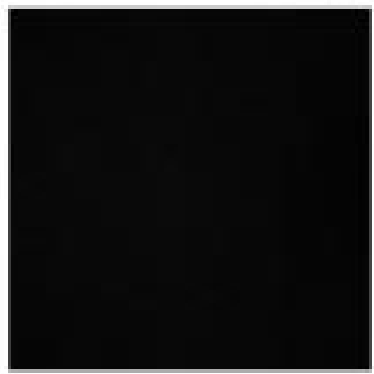

$-300$

$+150$
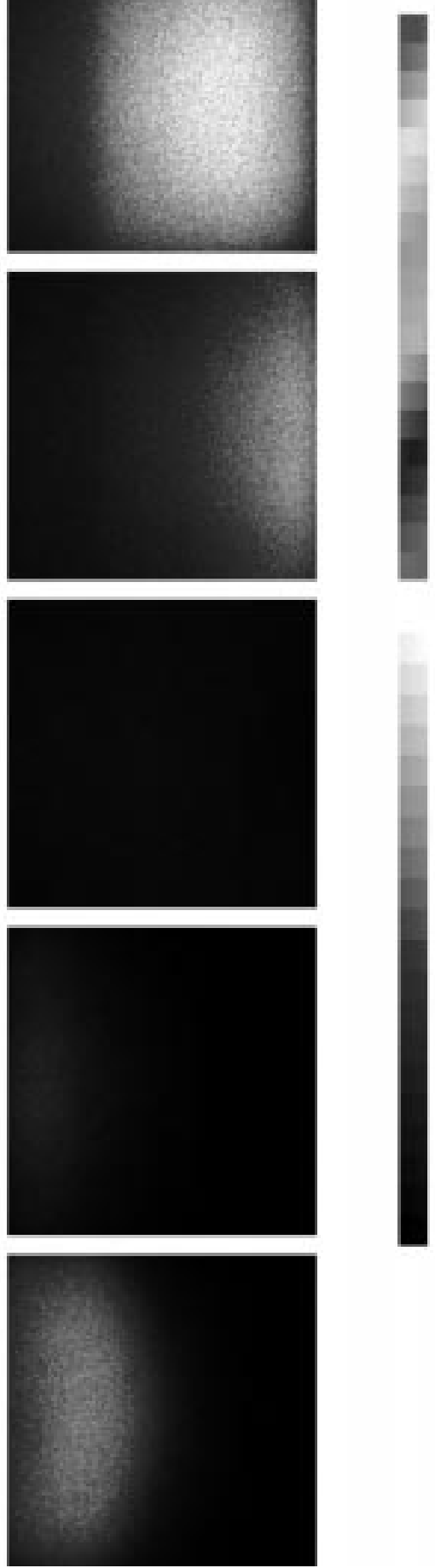

$+150$

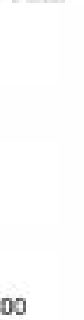

Fig. 7. States, $w_{\text {sum }}$ and pyramidal-pyramidal excitation for pyramidal neurons after weak shock stimulus. 

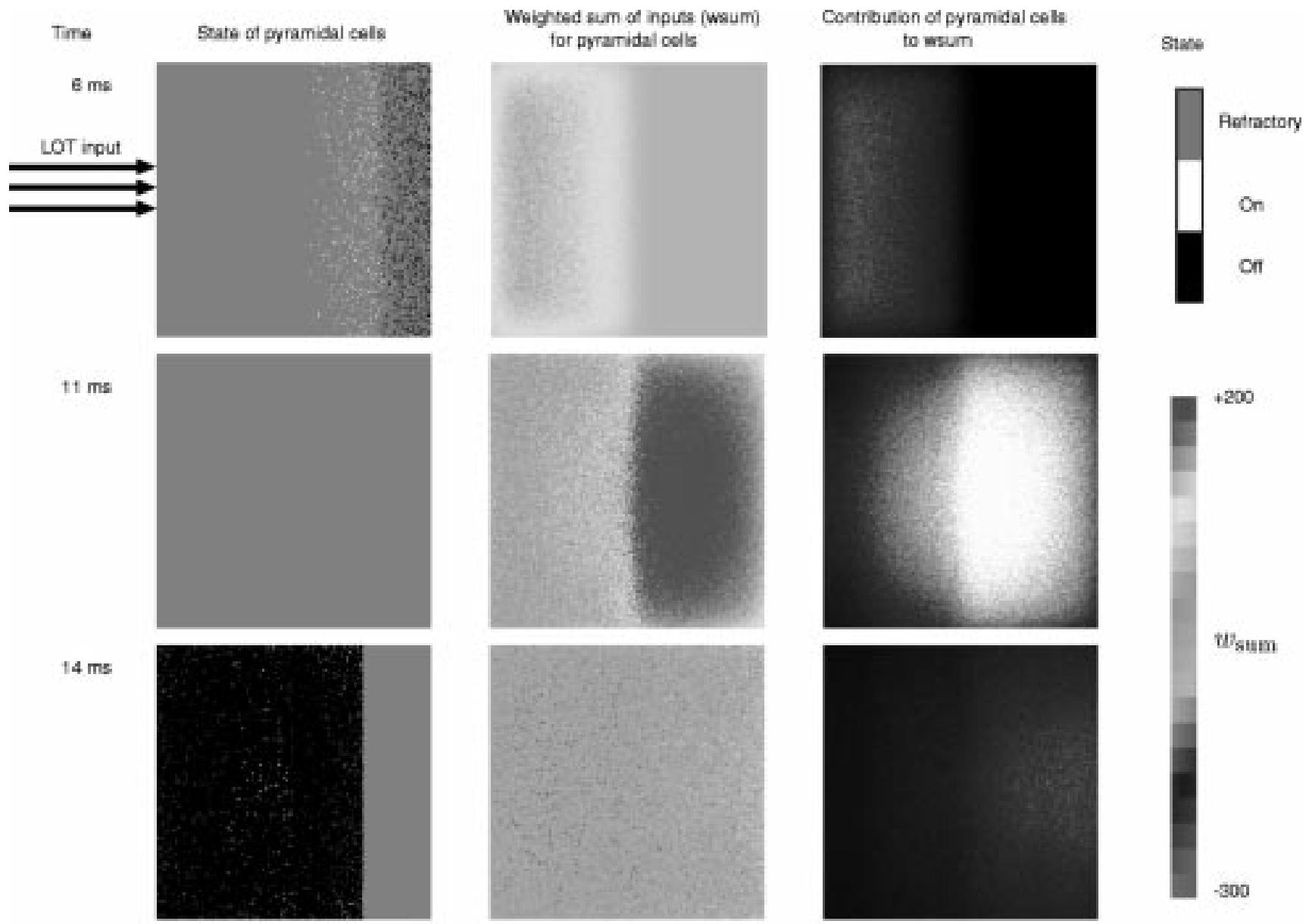

300
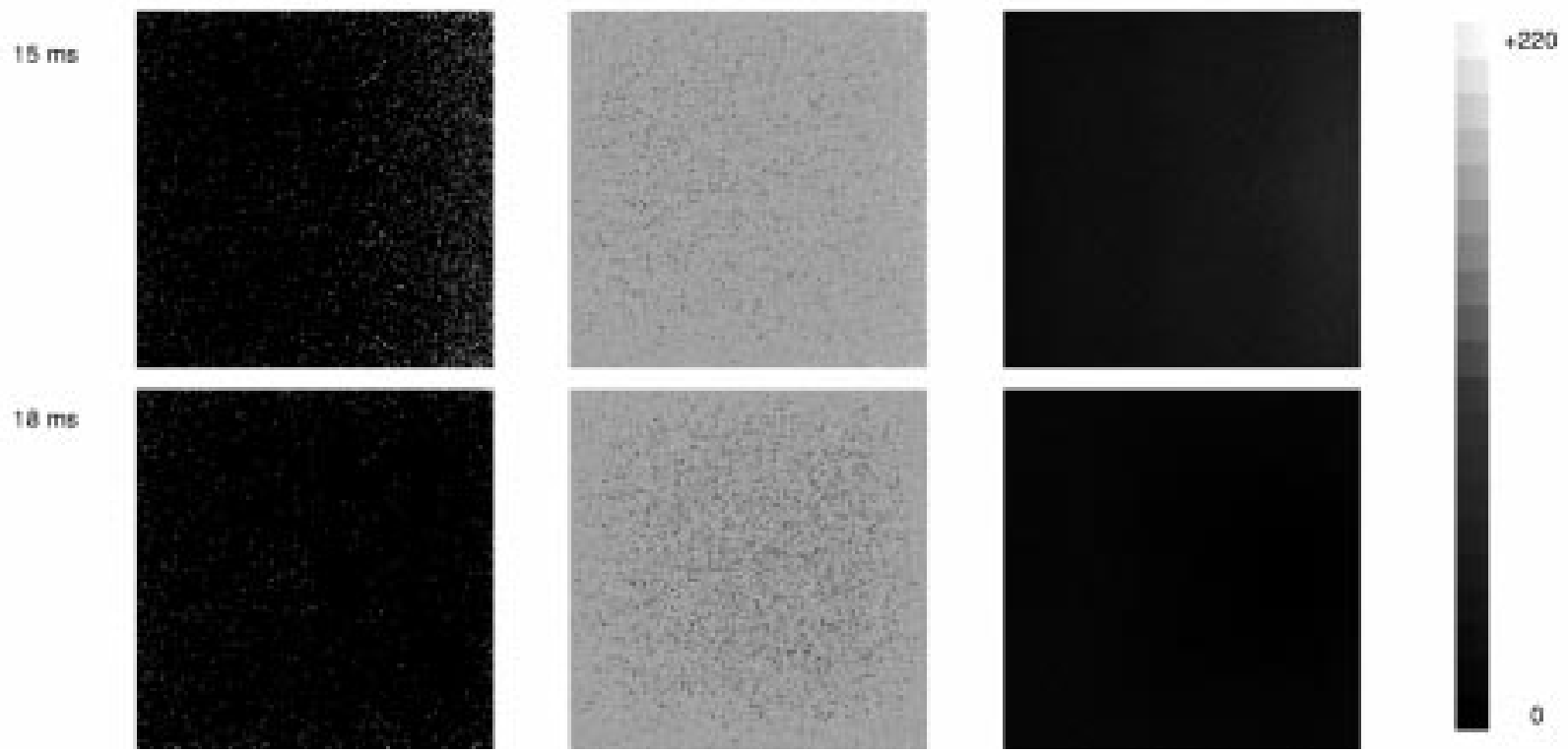

Fig. 8. States, $w_{\text {sum }}$ and pyramidal-pyramidal excitation for pyramidal neurons after strong shock stimulus.

Fig. 7 shows how the weak stimulus triggers a wave of activity, its front reaching the distant region of the cortex after $9 \mathrm{~ms}$ (leftmost top panel). The middle and right panels show a zone of excited cells (lighter region) generated by the passing wave. In the wake of the excitatory wave, appears a region of inhibited cells (darker pixels in the middle panels) due to the activation of inhibitory cells in the $G A B A_{A}$ and $G A B A_{B}$ layers. How- ever, at $t=29 \mathrm{~ms}$, the area where the first wave was originated (leftmost region in all panels) is returning to the initial state. The disappearance of inhibition makes it possible to generate a second wave, which has advanced up to the central region of the cortex at $t=33 \mathrm{~ms}$.

Fig. 8 shows the results for the high intensity shock stimulus. In comparison with Fig. 7, the initial wave of excitation propa- 


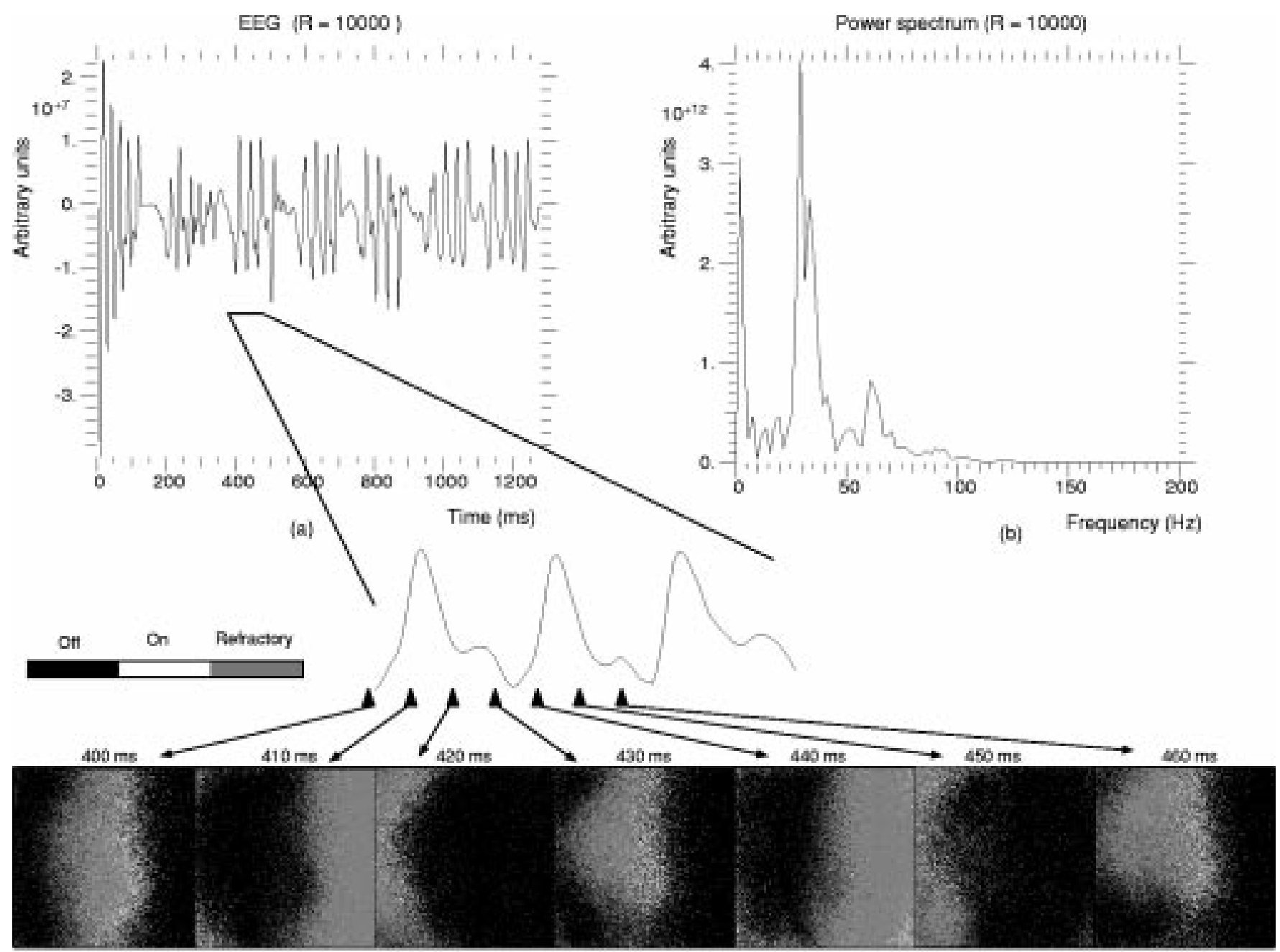

(c)

Fig. 9. (a) Simulated EEG. (b) Power spectrum of the EEG. (c) Cortical waves underlying EEG oscillations.

gates faster, arriving at the far end of the cortex at $t=6 \mathrm{~ms}$ (see leftmost top panel). However, at $t=14 \mathrm{~ms}$ and $t=16 \mathrm{~ms}$ the entire pyramidal layer receives inhibition following the passing wave (middle column) and intensifies at $t=18 \mathrm{~ms}$. Long-range excitatory connections between pyramidal cells (see rightmost column) are unable to generate a second wave. Simultaneously, the pyramidal to pyramidal excitation has also decreased (compare rightmost panels at $t=14-15 \mathrm{~ms}$ and $t=18 \mathrm{~ms}$ ). The remaining excitation is only able to trigger sparse action potentials in a few cells (see leftmost region in the first column of panels at $t=15-18 \mathrm{~ms}$ ).

In contrast with the weak stimulus, the strong stimulus causes fast excitation which leads to the disappearance of excitatory input before the GABA inhibition following the passing wave deactivates.

Note at $t=13 \mathrm{~ms}$ in Fig. 7 and at $t=14 \mathrm{~ms}$ in Fig. 8 the appearance of bands of refractory neurons. This artifact is caused by the quantization of the axonal propagation delays, an strategy that reduces memory consumption. It was observed during the initial wave after shock stimulus but disappeared in subsequent waves as the firing desynchronized.

\section{Random Input Response}

Experimentally recorded EEGs [21] and simulations with compartmental models [11] display clear oscillations (Fig. 5). These pseudo-periodic EEG profiles are thought to be supported by spatial waves of excitation [22], [23] sweeping across the cortex. To study these phenomena with our cortical model, a long-lasting random input stimulus was used. Random excitation is more closely related to the normally functioning piriform cortex than the shock stimulus. It was generated by spreading the firing times of the LOT axons throughout the entire simulation. Each neuron in the LOT pool was configured to fire once and its firing time is given by a uniform distribution in the range $\left(0-t_{\mathrm{stop}}\right)$, where $t_{\mathrm{stop}}$ is the duration of the simulation. Hence, the stimulus intensity, expressed as the average number of excitatory synaptic connections from LOT axons to pyramidal neurons activated per unit of time, is given by

$$
R=\frac{N_{\mathrm{LOT}} C_{\mathrm{LOT}-\mathrm{to}-\mathrm{pyr}}}{t_{\mathrm{stop}}}
$$




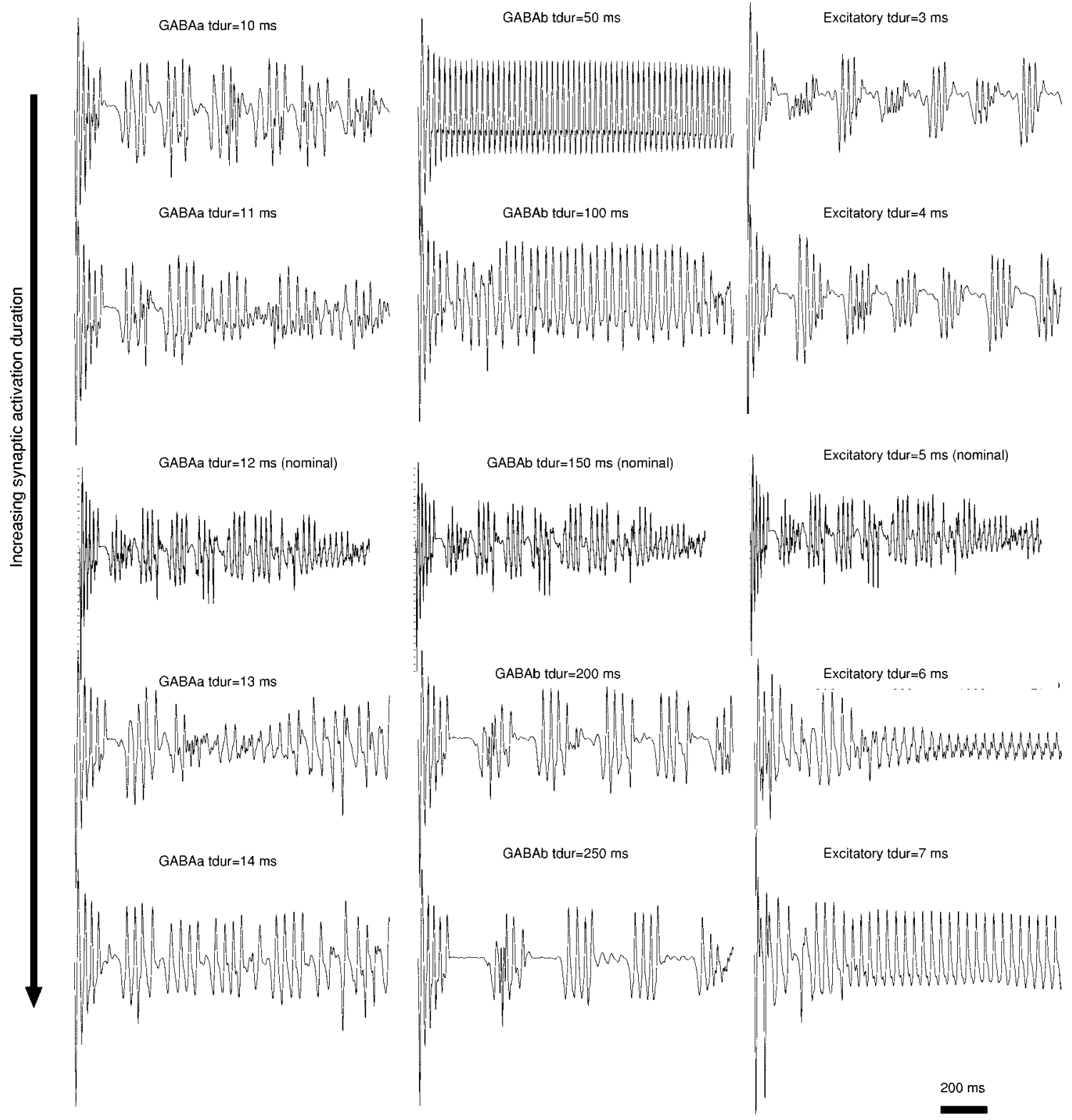

Fig. 10. EEG profile for several values of $t_{\mathrm{cur}}$ in $G A B A_{A}, G A B A_{B}$, and excitatory synapses.

where $N_{\mathrm{LOT}}$ is the number of LOT axons and $C_{\mathrm{LOT}}$-to-pyr the number of connections to pyramidal cells from a single LOT axon. Fig. 9(a) shows the simulated EEG obtained using a grid of $10 \times 10$ electrodes and a stimulus of $R=10^{4}$ activations $/ \mathrm{ms}$. Fig. 9 (b) is the corresponding power spectrum.

The unstructured random input generates a structured activity pattern in the cortical model. The simulated EEG [Fig. 9(a)] shows an initial transitory phase of approximately $150 \mathrm{~ms}$, followed by sequences of peaks with intervals of diminished activity. In its power spectrum [Fig. 9(b)], two main frequency bands appear; $0-10 \mathrm{~Hz}$ and a higher frequency range 30-35 Hz. A secondary harmonic peak is also present at $60-65 \mathrm{~Hz}$. Fig. 9(c) shows a state map of the pyramidal layer. It is characterized by cortex-wide excitation waves, reminiscent of those observed for shock stimulus. Each peak in the EEG is associated with a single wave propagating across the cortex.

\section{Effect of Synaptic Parameters on EEG Profiles}

The impact of model parameters on the characteristics of the EEG was explored. In particular, variations of $t_{\text {dur }}$ in inhibitory and excitatory synapses were found to trigger EEG profile transitions. Fig. 10 shows the EEG traces for several values of $t_{\text {dur }}$ in $G A B A_{A}$ (leftmost column), $G A B A_{B}$ (middle column), and excitatory synapses (rightmost column). The middle trace in all columns corresponds to nominal values. Variations in $G A B A_{A}$ synapses affects EEG regularity; see $t_{\mathrm{dur}}=14 \mathrm{~ms}$ compared to $t_{\mathrm{dur}}=10 \mathrm{~ms}$ and $t_{\mathrm{dur}}=11 \mathrm{~ms}$. A decrease of $t_{\mathrm{dur}}$ in $G A B A_{B}$ synapses from $150 \mathrm{~ms}$ to $50 \mathrm{~ms}$ leads to total absence of EEG bursts and nearly sinusoidal traces. Conversely, an increase to $250 \mathrm{~ms}$ results in an EEG with longer interburst latencies.

An opposite effect results from changes in the activation duration of excitatory synapses (see rightmost column in Fig. 10). For a value of $t_{\mathrm{dur}}=3 \mathrm{~ms}$ (top), the EEG corresponds to 
a biphasic sequence of bursts. Progressive increases (toward bottom) lead to a steady-state consisting of nearly sinusoidal profile, for $t_{\mathrm{dur}}=6 \mathrm{~ms}$ and $t_{\mathrm{dur}}=7 \mathrm{~ms}$.

\section{DISCUSSION}

A model of the piriform cortex has been constructed by means of a hierarchically defined finite-state automaton neuron. It aims at demonstrating the usefulness of an event-driven framework where some fundamental features of neuronal function can be captured while avoiding the computational complexity inherent to analog models. The model with $10^{5}$ neurons and $3 \times 10^{7}$ synapses represents an increase of two to three orders of magnitude in problem size with respect to previous simulations [11], [12]. The performance obtained was 0.9 s CPU time per simulated ms. To achieve a similar computational cost with analog techniques, the network size must be limited to hundreds or thousands of neurons, depending on neuron model complexity. Alternatively, high-cost parallel architectures must be employed.

The standard approach to realistic simulation of large neural aggregates makes use of an analog paradigm based on core-conductor theory of axons and dendrites [24] and Hodgkin-Huxley ion channel models [3]. Computational complexity can be reduced in various ways; minimizing the number of isopotential segments (compartments), limiting the number ion channel types or even substituting those responsible for action potential generation by a threshold function (e.g., the integrate and fire model [25]). Uncoupling of the equations belonging to different neurons is possible by exploiting the discrete nature of the spike. In this case, analog models describe neurons whereas an event-driven engine manages the inter-neuron communication at the synaptic level [4].

We have used an abstract event-driven description of the neuron, eliminating the need for a continuous simulation engine in exchange for the resulting loss of realism. Within this framework, computational efficiency arises from the state-update scheme, where only those neurons receiving messages at a particular time point must be re-evaluated. The simplicity of the update operation also contributes to diminishing the need for processing resources.

To investigate the dynamics of a large-scale cortical model, the cell types and the connectivity patterns in the network were constrained by piriform cortex anatomy. The three synaptic classes considered, $G A B A_{A}, G A B A_{B}$ and excitatory were configured with relative efficacies and time constants in accordance with experimental findings. The following issues have been addressed: 1) wave generation in a pyramidal layer deprived of inhibition; 2) response of the model, including pyramidal and inhibitory inter-neurons, to pulse-like excitation (shock stimuli); 3) the genesis of EEG oscillations as a result of unpatterned long-lasting stimuli; and 4) the modulation of the temporal EEG profiles by variations in the synaptic time constants.

\section{A. Shock Stimulus}

The predicted response to shock stimuli shows nonlinear properties in agreement with experimental results [10]. Namely, a low intensity pulse stimulus elicits a sequence of model-wide excitation waves and a ringing EEG whereas high intensity stimuli lead to single waves and single peak EEGs. More subtle experimental results are also accounted for by the model. For instance, current source density analysis shows that different synaptic types are maximally active at different points in time during shock response [26]. This effect can be seen in Fig. 6(a) where two secondary peaks can be distinguished, the first corresponding to excitatory synapses from LOT to pyramidal neurons and the second to the delayed activation of pyramidal to pyramidal synapses. Similar double-bumped shock responses were obtained with compartmental [11] and relaxation models [22].

\section{B. Random Stimulus}

Waves of excitation have been proposed as the physical phenomena underlying EEG oscillations and are thought to arise throughout the cortex [23]. Experimental in vivo studies of piriform cortex and olfactory bulb activity have indeed confirmed that EEG oscillations occur and are especially regular during odour inhalation [27].

To investigate the generation of cortical waves, a temporally unstructured input stimulus was used. The axons of the LOT, which project to the piriform cortex from the olfactory bulb in the actual cortex, were configured to fire randomly throughout the simulation. This setup aimed at producing an input stimulus more closely related to the real pattern of activations than that utilized in shock experiments. Its random nature guarantees that the derived cortical spatio-temporal patterns arise from the intrinsic anatomical and dynamical properties of the model rather than the pre-arranged structure of its input.

This activity leads to cortex-wide waves in our model, in line with compartmental simulations [11]. Further, each EEG peak can be related to a particular cortical wave sweeping across the model. These waves are preferentially originated in the leftmost region in all panels (corresponding to the rostral end in the actual cortex). This result is the consequence of the decrease of LOT-pyramidal connections when moving from left to right in the panels (rostral to caudal in the actual cortex), an anatomical feature already observed in early experimental studies [28].

There is experimental and theoretical evidence supporting the hypothesis that global changes of network parameters trigger a switch between functionally different aggregate states. In the olfactory cortex, such a mechanism has been reported [12], [29]. The generalized release of acetylcholine is thought to alter synaptic dynamics and neuronal excitability [30], triggering a mode transition from memory recall to memory acquisition. More generally, abrupt EEG transitions are a well known phenomenon, often associated with changes of conscious states (sleep, walk, anesthesia and so on).

The variations in the temporal and frequency profiles of the EEG resulting from parameter changes were investigated. In particular, transitions between nearly periodic, bursting and irregular EEGs can be achieved by means of changes in the synaptic activation duration $\left(t_{\mathrm{dur}}\right)$ of the different synaptic types. The emergence of spatially coherent patterns and the transition between modes of operation by means of network 
parameter variation is also in agreement with previous cortical simulations utilizing integrate and fire models [31].

Additional simulations were carried out with equal size cell populations, with three $150 \times 150$ grids corresponding to the pyramidal, fast and slow inhibitory cell types (results not shown). This was done to compare our results with those obtained by Wilson et al. [11] who included 1500 neurons in each pool. With this configuration, a parameter set was also found which produced single peak and damped ringing in response to shock stimuli and continuous oscillations in response to unpatterned long-lasting input. In the latter case, the main spectral component was centered at $40 \mathrm{~Hz}$, which coincides with the results in [11]. This constitutes a shift of approximately $10 \mathrm{~Hz}$ toward higher frequencies with respect to the spectra described in the results section, obtained for more realistic relative population sizes. The large parameter space presented by the model makes it likely that multiple configurations exist whose EEG share a number of temporal and frequency characteristics. As already shown, the synaptic parameter $t_{\mathrm{dur}}$ affects EEG profiles and suggests that parameter tuning would induce further shifts in the main frequency components.

The simulations carried out have aimed at comparing the model response with experimentally obtained recordings and theoretical investigations with analog neuron descriptions. It has not been attempted, however, to link network dynamics to the suspected functionality of the piriform cortex within the olfactory system. Several studies have tackled this problem: the Lynch-Granger model [32], [33] suggests that the system olfactory bulb-olfactory cortex performs hierarchical clustering of the cue environment; the Li-Hertz model [14] proposes that odour recognition is achieved by a resonance phenomenon between cortex and bulbar oscillations; the Wilson-Bower model [34] implements an associative memory able to store and retrieve odour information.

A feature shared by these models is the use of synaptic modification algorithms or other types of network plasticity which must be activity-dependent to allow the storage of new odours. The model described here does not incorporate plasticity. However, the striking similarities between the physiological data and the results obtained with the simple event-driven model supports the possibility of utilizing the same framework for investigations of the functional role of synaptic plasticity.

\section{CONCLUding REMARKS}

In summary, the approach presented in this paper allows large-scale neural simulations on single processor desktop computers and the exploration of the effects of physiological parameters on neural population dynamics. This is achieved by devising a completely event-driven framework where the need for computationally costly continuous simulation engines is eliminated altogether.

The piriform cortex model provides a tool for the testing of theories related to the nature of the computations carried out by cortical structures. The results obtained so far demonstrate that the main features of the response by the olfactory cortex to short and long-lasting stimuli can be accounted for by a simple event-driven cortical model. However, there is some experimental and theoretical evidence that mechanisms other than those described here may also lead to oscillatory activity in cortical structures; the afferents from the olfactory bulb may carry temporally structured activity, causing synchronized cortical dynamics [10] and there is evidence that respiratory rythms correlate with oscillations under anesthesia (Fontanini et al., unpublished work), indicating that extracortical sources, instead of intracortical circuits, may actually support oscillations.

The techniques presented here can also be applied to other areas of the nervous system. The reduction of computational complexity in comparison with alternative strategies suggests the adequacy of the proposed approach for large-scale models incorporating multiple cortical areas. Research is underway to exploit Beowulf clusters in order to provide the necessary resources for such computationally costly simulations. Preliminary results indicate that problem size could be increased at least one order of magnitude by means of a proportional increase in the number of processing nodes with a mere $10 \%$ simulation time overhead due to internode communication.

\section{REFERENCES}

[1] C. Koch and I. Segev, Eds., Methods in Neural Modeling: From Ions to Networks. Cambridge, MA: MIT Press, 1998.

[2] J. A. Freeman and D. M. Skapura, Eds., Neural Networks: Algorithms, Applications and Programming Techniques. Reading, MA: AddisonWesley, 1992.

[3] A. L. Hodgkin and A. F. Huxley, "A quantitative description of membrane current and its application to conduction and excitation in nerve," J. Physiol., vol. 117, pp. 500-544, 1952.

[4] P. Hammarlund and O. Ekeberg, "Large neural network simulations on multiple hardware platforms," J. Computational Neurosci., vol. 5, pp. 443-459, 1998.

[5] J. M. Bower and D. Beeman, Eds., The Book of Genesis. Berlin, Germany: TELOS/Springer-Verlag, 1998.

[6] M. L. Hines and N. T. Carnevale, "The neuron simulation environment," Neural Computation, vol. 9, no. 6, 1997.

[7] E. Pytte, G. Grinstein, and R. D. Traub, "Cellular automaton models of the CA3 region of the hippocampus," Network: Computation Neural Syst., vol. 2, no. 2, pp. 149-167, 1991.

[8] R. Mansharamani, "An overview of discrete event simulation methodologies and implementation," Sadhana, vol. 22, no. 5, pp. 611-627, 1997.

[9] L. B. Harberly and S. L. Feig, "Structure of the piriform cortex of the opossum. I. Fine structure of cell bodies and neuropil," J. Computational Neurol., vol. 216, pp. 69-88, 1983.

[10] K. L. Ketchum and L. B. Haberly, "Synaptic events that generate fast oscillations in piriform cortex," J. Neurosci., vol. 13, no. 9, pp. 3980-3985, 1993.

[11] M. Wilson and J. M. Bower, "Cortical oscillations and temporal interactions in a computer simulation of piriform cortex," J. Neurophysiol., vol. 67, no. 4, pp. 981-995, 1992.

[12] E. Barkai, R. E. Bergman, G. Horwitz, and M. E. Hasselmo, "Modulation of associative memory function in a biophysical simulation of rat piriform cortex," J. Neurophysiol., vol. 72, no. 2, pp. 659-677, 1994.

[13] L. B. Harberly and J. M. Bower, "Olfactory cortex: Model circuit for study of associative memory?,” TINS, vol. 12, no. 7, pp. 258-264, 1989.

[14] Z. Li and J. Hertz, "Odor recognition and segmentation by coupled olfactory bulb and cortical networks," Neurocomputing, vol. 26-27, pp. 789-794, 1999.

[15] E. T. Claverol, A. D. Brown, and J. E. Chad, "Discrete simulation of large aggregates of neurons," Neurocomputing, vol. 47, pp. 277-297, 2002.

[16] R. L. Bagrodia, K. M. Chandy, and J. Misra, "A message-based approach to discrete-event simulation," IEEE Trans. Software Eng., vol. SE-13, pp. 654-665, Dec. 1987.

[17] W. Rall, "Electrophysiology of a dendritic model," Biophys. J., vol. 2, pp. 145-167, 1962

[18] M. Klee and W. Rall, "Computed potentials of cortically arranged populations of neurons," J. Neurophysiol., vol. 40, pp. 647-666, 1977. 
[19] W. H. Press, W. T. Vetterling, S. A. Teukolsky, and B. P. Flannery, Numerical Recipes in $C$, 2nd ed. Cambridge, U.K.: Cambridge Univ. Press, 1992, ch. 13.

[20] W. J. Freeman, "Relations between unit activity and evoked potentials in prepyriform cortex in cats," J. Neurophysiol., vol. 31, pp. 337-348, 1968.

[21] S. L. Bressler, "Spatial organization of EEG's from olfactory bulb and cortex," Electroencephalogr. Clin. Neurophysiol., vol. 57, pp. 270-276, 1984.

[22] T. Ballain, P. Litaudon, J. Martiel, and M. Cattarelli, "Role of the net architecture in piriform cortex activity: Analysis by a mathematical model," Biological Cybern., vol. 79, no. 4, pp. 232-336, 1998.

[23] P. L. Nunez, Electric Fields of the Brain: The Neurophysics of EEG. New York: Oxford Univ. Press, 1981.

[24] W. Rall, "Core conductor theory and cable properties of neurons," in Handbook of Physiology, E. R. Kandel, Ed. Bethesda, MD: American Physiological Society, 1977, pp. 39-97.

[25] W. Gerstner, "Time structure of the activity in neural network models," Physical Rev. E, vol. 51, pp. 738-758, 1995.

[26] K. L. Ketchum and L. B. Haberly, "Membrane currents evoked by afferent fiber stimulation in rat piriform cortex I. Current source-density analysis," J. Neurophys., vol. 69, no. 1, pp. 248-260, 1993.

[27] W. J. Freeman, "The physiology of perception," Amer. Scientif., vol. 264, no. 2, pp. 78-85, 1991.

[28] J. E. Schwob and J. L. Price, "The cortical projection of the olfactory bulb: Development in fetaland neonatal rats correlated with quantitative variations in adult rats," Brain Res., vol. 151, pp. 369-374, 1978.

[29] M. E. Hasselmo and E. Barkai, "Cholinergic modulation of activity-dependent synaptic plasticity in the piriform cortex and associative memory function in a network biophysical simulation," J. Neurosci. vol. 15 , no. 10 , pp. $6592-6604,1995$.

[30] E. Barkai, G. Horwitz, R. E. Bergman, and M. E. Hasselmo, "Modulation of the input/output function of rat piriform cortex pyramidal cells," J. Neurophysiol., vol. 72, no. 2, pp. 644-658, 1994.

[31] S. L. Hill and A. E. P. Villa, "Dynamic transitions in global network activity influenced by the balance of excitation and inhibition," Network: Computation Neural Syst., vol. 8, no. 2, pp. 165-184, 1997.

[32] J. Ambros-Ingerson, R. Granger, and G. Lynch, "Simulation of paleocortex performs hierarchical clustering," Science, vol. 247, pp. $1344-1348,1990$

[33] E. Barnard, "Analysis of the Lynch-Granger model of olfactory cortex," Biological Cybern., vol. 62, no. 2, pp. 151-155, 1989.

[34] M. Wilson and J. Bower, "A computer simulation of olfactory cortex with functional implications for storage and retrieval of olfactory information," in Neural Information Processing Systems, D. Z. Anderson, Ed. New York: American Institute of Physics, 1988, pp. 114-126.

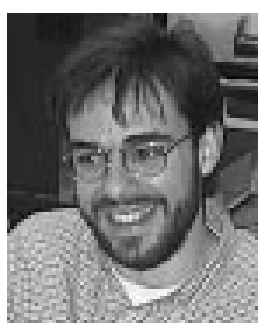

Enric T. Claverol received the B.Sc. degree (with honors) in telecommunications engineering (with honors) from the Polytechnic University of Catalonia, Catalonia, Spain in 1997 and the Ph.D. degree in computational neuroscience from the University of Southampton, Hampshire, U.K., in 2000.

He is currently a Postdoctoral Research Fellow at the California Institute of Technology, Pasadena, CA. His research interests include simulation of neural aggregates, multielectrode arrays for the study of development in in vitro cultures, and novel brain imaging technologies.

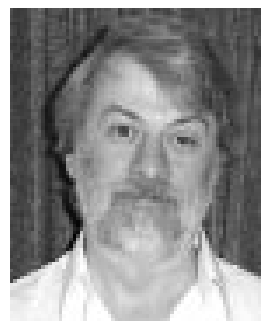

Andrew David Brown (M'90-SM'96) received the B.Sc. degree (with honors) in physical electronics and the Ph.D. degree in microelectronics from Southampton University, Hampshire, U.K., in 1971 and 1981, respectively.

He was appointed Lecturer in Electronics at Southampton University in 1981, Senior Lecturer in 1989, and Reader in 1992. He was appointed to an established chair of Electronics in 1998. He was a Visiting Scientist at IBM, Hursley Park, U.K., in 1983 and a Visiting Professor at Siemens NeuPerlach, Munich, Germany, in 1989. He is currently Head of the Electronic System Design Group, Electronics Department, Southampton University. The group has interests in all aspects of design automation: simulation, modeling, synthesis, and testing.

Prof. Brown is a Fellow of the Intitution of Electrical Engineers, a Chartered Engineer, and a European Engineer

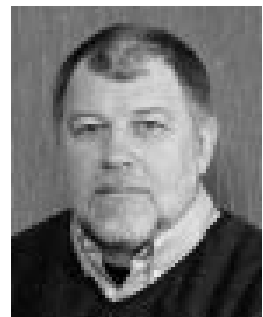

John Edward Chad received the B.Sc.degree (with honors) in physiology and biochemistry and the Ph.D. degree in neurophysiology from the University of Southampton, Hampshire, U.K., in 1974 and 1978 , respectively.

He worked as a Postdoctoral Research Fellow (1978-1982) and Faculty Assistant Research Biologist (1983-1985) at the University of California, Los Angeles, before taking up the post of Senior Scientist at the Sandoz Institute for Medical Research, London, U.K. (1985-1986). He was appointed as a Lecturer in the School of Biological Sciences, Southampton University in 198 and is currently Senior Lecturer in Neuroscience. His research interests include neurocomputation, control of neuronal excitability, and neurodegeneration. 